\title{
Los desafíos de la construcción identitaria del Estado para la paz: una aproximación desde el análisis del discurso
}

\section{The Challenges of the State Identity for Peace: An Approach from Discourse Analysis}

\author{
Erika M. Rodríguez Pinzón ${ }^{1}$ \\ Universidad Autónoma de Madrid (España)
}

Recibido: $30-10-17$

Aprobado: 22-01-18

\section{Resumen}

El Estado colombiano ha sido construido alrededor del conflicto armado y de la violencia política que ha marcado la historia del país. De hecho, más que un Estado a pesar de la guerra, podría decirse que el colombiano es un Estado de la guerra. Esto supone una paradoja y un desafío para la construcción de la paz, dado que implica desarraigar y poner en entredicho muchos de los conceptos y construcciones identitarias sobre las que se articulaba el discurso político y una buena parte de la estructura de la política en Colombia. Este artículo tiene como objetivo analizar la forma en la que en el discurso político se ha articulado la imagen del Estado de cara a los desafíos que supone la paz. Para ello se aplica una metodología basada en la identificación de los discursos básicos sobre los que se construye la "autorepresentación" del Estado y en estudiar sus características y paradojas frente al proceso de implementación del proceso de paz.

Palabras-clave: Colombia, paz, análisis del discurso, relaciones internacionales.

\footnotetext{
${ }^{1}$ (erika.rodriguez@uam.es ). Doctora en Relaciones Internacionales de la Universidad Autónoma de Madrid. Profesora Asociada de la Facultad de Derecho (Departamento de Relaciones Internacionales) de la Universidad Autónoma de Madrid y de la Universidad Internacional de La Rioja - UNIR, España 


\begin{abstract}
The foundations of the Colombian State are linked to the development of internal conflict and political violence that have marked the history of the country. This situation creates a huge challenge for peace consolidation since it involves questioning and scrapping many of the concepts and identity frameworks over which political discourses and structures have been built in Colombia.

The objective of this article is to analyze the way the image of the State has been articulated in the Colombian political discourses and how it has been affected by the recent Peace Process.
\end{abstract}

Key-words: Colombia, Peace, Discourse Analysis, International Relations.

\title{
1. Introducción
}

Este artículo tiene como fin aprovechar la teoría de Relaciones Internacionales para hacer un análisis de los elementos constitutivo del discurso sobre el Estado en Colombia en lo que respecta a la forma en la se ha construido históricamente y en las transformaciones que se esperan del mismo de cara a la efectividad el proceso de postconflicto.

Basados en una aproximación postestructuralista, hay dos facetas fundamentales de la identidad que pueden estudiarse. Por un lado, los que tienen que ver con la definición del Estado, es decir, la forma en la que los gobernantes lo definen y las paradojas que suscita esta definición frente a un escenario de postconflicto. Por el otro lado, aquellos que tratan la identidad relacional, esto es, la forma en la que se define frente a los otros y más concretamente la forma en la que categoriza dicha diferenciación entre pares, amigos y enemigos (Campbell, 1998).

En este último caso también hay un desafío importante dado que se supone que en el nuevo escenario el enemigo por antonomasia, las guerrillas se convierten en actor político. Por primera vez en la política colombiana, el espacio de la participación política debería acoger la propuesta política guerrillera que según parece extraerse del reciente Congreso, del ahora movimiento político, conserva varios de sus preceptos ideológicos más revolucionarios y, por tanto, alejados de los discursos que han determinado el espacio electoral del país.

Este análisis parte de la forma en la que se ha estructurado el discurso oficial y gubernamental, para enfrentarlo a las paradojas y desafíos que supone el escenario de postconflicto. Se centra en la primera faceta de la construcción de la identidad, es decir en sus discursos básicos, o pilares de autoidentificación. Asimismo, y aunque no incide en la identidad relacional, rescata algunos rasgos de la misma. 


\section{La construcción de la identidad del Estado}

Recientemente el Presidente Juan Manuel Santos respondía al presidente de Venezuela Nicolás Maduro, por referirse éste a Colombia como un "Estado fallido". Así, Santos afirmó que un Estado fallido "es considerado un Estado que no controla el territorio" y que en otras épocas estuvimos "ad portas de serlo, debido al narcotráfico". Más aún, el presidente recordó que entre los elementos necesarios para no ir camino de ser una Estado fallido estaba la búsqueda de la paz.

Esta breve afirmación sirve de ejemplo para iniciar el análisis de la construcción discursiva del Estado en Colombia. En primer lugar, se evidencia lo que ya ha sido una constante entre los tres últimos mandatarios que ha tenido el país, la idea de que el país estuvo ad portas de ser un Estado fallido y que dicha historia se cambió totalmente.

Numerosos trabajos académicos han estudiado el caso de Colombia desde el punto de vista de los estados fallidos. Algunos de forma muy temprana como Paul Oquist (1978), aunque la mayoría se realizaron en la primera década del siglo XXI, cuando la situación de violencia en el país por cuenta de la confrontación entre guerrilleros, paramilitares y gobierno alcanzó cotas históricas. Ciertamente la mayor parte de los trabajos coinciden en señalar el "colapso parcial del Estado" (Bejarano y Pizarro, 2003; Mason, 2002) o la particularidad de la debilidad del Estado en Colombia (González, Bolívar y Vásquez, 2003) y de la convivencia entre violencia y orden (Gutiérrez, 014).

La reflexión que se propone en este artículo no incide en el análisis académico de las características de las fallas del Estado, sino que se centra en el discurso político y la forma en la que se autodefine el Estado. Tal como lo sostiene Juan Gabriel Tokatlian (2008), desde el ángulo de los tomadores de decisión, un "Estado fallido" no era un Estado hostil y pendenciero, sino una mezcla de Estado inepto e indolente; lo cual lo hacía ingobernable. En este sentido los "Estados fallidos" se diferencian claramente de lo que después del $11 \mathrm{~S}$ se denominaran los "rogue states", que más que no tener la capacidad para contener una amenaza a la seguridad, son una amenaza en sí mismos porque su voluntad política se contrapone de forma abierta y beligerante a los valores determinados por las potencias occidentales.

En 1997 los documentos estratégicos de la seguridad estadounidense apuntaban que los Estados fallidos o en proceso de serlo podían ser fuente de inestabilidad, conflictos internos o crisis humanitarias en zonas en las que se encuentran intereses de Estados Unidos. Tras los atentados del año 2001, los temores encontraron una expresión real y demandaron mayor cantidad de recursos para atender la amenaza. De hecho, el Segundo Informe de Seguridad

${ }^{2}$ El Tiempo 22/04/2017 Presidente Santos respondió las críticas de Nicolás Maduro

Araucaria. Revista Iberoamericana de Filosofía, Política y Humanidades, año 20, n 39. Primer semestre de 2018. Pp. 491-507. ISSN 1575-6823 e-ISSN 2340-2199 doi: 10.12795/araucaria.2018.i39.23 
Nacional de Estados Unidos, presentado al Congreso por los senadores Gary Hart (demócrata) y Warren Rudman (republicano) en 2001, señalaba a México, Colombia, Rusia y Arabia Saudita, entre otros, como países en los que Washington tenía un especial interés en preservar la estabilidad doméstica.

La generación de un discurso de seguridad, y de su política en este sentido resulta muy interesante el análisis que sugiere Tokatlian y refuerzan otros autores colombianos como Socorro Ramírez (2001), Arlene Tickner (2001), Ricardo Vargas (1999), Sandra Borda (2012) o Diana Rojas (2006), para quienes la relación entre Estados Unidos y el Gobierno del presidente Samper (1994-1998) no solo fortaleció la narrativa del Estado fallido sino que de hecho debilitó al Estado colombiano en cuanto le impuso una marca a nivel internacional a la vez que alentó una crisis interna que mermó aún más la capacidad del gobierno.

Por su parte los expresidentes Pastrana (1998-2002) y Uribe (20022010) usaron esta idea durante sus mandatos para legitimar sus políticas de seguridad y de mano dura como una necesidad excepcional para corregir el destino del país (Rodríguez; 2017). Santos, en su caso, y a pesar de la distancia política que mantiene con su antecesor, construye sobre la misma idea y redunda en su beneficio, convirtiendo a la paz en una forma de evitar la falla estatal. Concretamente, incide en la comprensión del Proceso de Paz y de las implicaciones del Acuerdo de Paz como el coste final de superar el camino del fracaso.

Lo cierto es que, ni antes ni ahora, Colombia tiene un Estado que controle la totalidad del territorio y aunque la reducción de la violencia ligada al conflicto armado opera a favor de moderar las críticas sobre la incapacidad del Estado y ratifican la necesidad del Acuerdo de Paz, su capacidad operativa, legitimidad y representatividad distan de los mínimos necesarios para considerar que hay un control total del territorio. Es por este motivo que uno de los mayores desafíos del postconflicto son los territorios que ya no están bajo el control militar de las FARC. El gobierno mismo sabe que dichas zonas no pasan de inmediato al control del gobierno, por el contrario, son espacio de disputa de otros actores como el ELN o grupos paramilitares. Así, el presidente reconocía que

-"Hemos visto cómo en diferentes zonas del país los grupos armados ilegales están queriendo llenar los espacios que las FARC han venido dejando en su movilización hacia los puntos a donde se están concentrando" ${ }^{3}$.

Desde mediados de los noventa la posibilidad de convertirse, o más aún de ser señalado como un Estado fallido, es una de las mayores preocupaciones

${ }^{3}$ Mimi Yagoub; 24 de noviembre de 2016: Santos reconoce que grupos criminales se están trasladando a áreas de las FARC; Insight Crime; Disponible en: http://es.insightcrime.org/analisis/ santos-reconoce-grupos-criminales-estan-trasladando-areas-farc 
de los presidentes del país. En parte por el descredito que ello supone para un país que se considera, moderno, democrático e incluso modélico políticamente en la región. En segundo lugar, por lo que ello supone para la legitimidad de las élites en su relación con sus referentes globales. En una democracia altamente marcada por el carácter elitista de la clase política, la falla del Estado se convierte en un deslegitimizador de un orden social de privilegios basado en la estabilidad y el control. La élite se entiende y se explica a sí misma como una garantía de orden. Si dicho orden se demuestra inexistente, sus privilegios son insostenibles (Rodríguez, 2017).

Este breve ejemplo del discurso gubernamental da paso a un análisis más profundo de las características y pilares sobre el que se construye y en su relación con las expectativas y necesidades de un periodo efectivo de postacuerdo y postconflicto.

\section{La metodología postestructuralista}

El objeto de este artículo no es desarrollar in extenso una formulación metodológica, sino analizar los resultados de su aplicación sobre el caso en cuestión. El análisis se articula sobre la idea de los "discursos básicos", una herramienta tomada del postestructuralismo, como pilares sobre los cuales se construye un discurso en el cual se traducen los elementos más importantes referentes de la identidad del formulador del discurso.

En general puede decirse que la teoría del discurso postestructuralista examina cómo los textos escritos y los discursos son fenómenos constructivos, que forman las identidades y prácticas de los sujetos humanos. Particularmente, los discursos básicos son construcciones analíticas que ofrecen un tipo ideal de las dimensiones espacial, temporal y ética de la identidad: pueden construir un "otro" y un "sí mismo", así como los diversos niveles de diferenciación con el otro y la articulación temporal, ética y espacial de la identidad (Hansen, 2006). Es decir, son los que produce un mismo actor que trata de distanciarse de su propio pasado o que utiliza un "otro" para construirse una identidad por oposición. Así, el presidente Santos señalaba hace unos años que: "el país estuvo "a punto de ser un Estado fallido", pero ha devenido una nación
"totalmente diferente", reconocida como "líder en crecimiento" e inmersa en
un proceso de paz"4.

${ }^{4}$ 21/11/2014 Revista Semana: Santos: "Colombia es hoy un país líder que evitó ser un Estado fallido" Disponible en: http://www.semana.com/nacion/articulo/juan-manuel-santos-en-foro-enestados-unidos-colombia-es-hoy-un-pais-lider-que-evito-ser-un-estado/411900-3 
Es decir, para señalar una diferenciación entre el país que era, y el que es actualmente.

En el apartado anterior ya se utilizó un ejemplo de como el discurso gubernamental contemporáneo en Colombia se aplica en distanciarse del país que era Colombia, para hacer referencia a una Colombia nueva y una Colombia que será en el futuro cuando se haya conseguido la paz.

En este sentido, los discursos básicos que se identifican implican más que una estrategia, también implican una relación activa con el exterior, un intercambio de mutuos reconocimientos y de caracterizaciones propias y de otros en el marco de un análisis estratégico de la correlación y de los objetivos materiales a alcanzar.

A partir del análisis de las características de las "fallas del estado" y de su aplicación en el caso de Colombia, se identifican los discursos básicos de los presidentes Pastrana, Uribe y Santos, y en especial tres pilares sobre los cuales se construyen las características autorreferenciales sobre el estado en Colombia: 1) el Estado se define como un sistema institucional completo y autosuficiente; 2) el Estado es depositario de la voluntad popular y por tanto legítimo; y finalmente 3) el Estado es una víctima de actores ajenos y arrogantes que no le han permitido desarrollarse y ejercer correctamente su papel.

Estos pilares, a su vez y según el análisis que se desarrollan determinan de un modo concreto las premisas de la actuación gubernamental. En primer lugar, la idea de la seguridad como fin y como medio para conseguir el desarrollo y, en segundo lugar, lugar la concepción del bienestar como objeto de la acción estatal bajo los preceptos del Estado liberal. Dado que el proceso y Acuerdo de paz ha desplazado una parte de la primera de estas premisas (al darla tácitamente por cumplida en lo que respecta a las FARC), en este artículo nos centramos en la caracterización del Estado.

\section{Colombia como Estado moderno y eficiente}

Uno de las características más importantes del discurso es la forma en la que se hace referencia al Estado colombiano, como institución y como concepto. El discurso se articula sobre la legitimidad y la capacidad existente y desarrollada del Estado para cumplir sus funciones. Se critican fallos del modelo político y de la estructura de funcionamiento burocrático, pero siempre se resalta la idea de un estado unitario, fuerte y moderno. De hecho, en el discurso del presidente y de las instituciones el Estado es un producto terminado, cuyos fallos son fallos de sus agentes, mas no de la estructura del mismo, y en ese sentido se equipara a los países desarrollados o se le señala como un ejemplo para otros. 
Es cierto que Colombia da muestra de unas instituciones bastante fuertes, un manejo bastante ortodoxo de la macroeconomía, sin grandes presiones inflacionarias, y un amplio consenso entre las elites y la dirigencia en cuanto a la deseabilidad de la democracia (González y Otero, 2006). A pesar de ello, los supuestos del Estado fuerte, plenamente desarrollado y dotado de instituciones eficaces y dirigidas por tecnócratas, que se plantean a nivel global, contrastan con la historia de la construcción del Estado-nación. La historia de la construcción y ampliación del Estado-nación que en el particular caso de Colombia tiene particularidades muy especiales.

Desde tiempos de la colonia española, el territorio colombiano nunca pudo constituirse como una unidad administrativa y de poder. La independencia y la formación de los gobiernos republicanos tampoco conseguirían la unidad nacional, en parte por la geografía y la estructura social de poblamiento, con largas extensiones de difícil acceso y bajísima concentración demográfica $\mathrm{y}$, por otra parte, porque la distribución del poder entre los partidos liberal y conservador desde su aparición en el siglo XIX, se encargaron de dividirse el control del territorio. Nueve guerras civiles entre partidos profundizaron la fragmentación del control político. Adicionalmente, a causa de la estructura geográfica, las amplias zonas despobladas y la ausencia de reformas agrarias que permitieran un acceso a los recursos productivos menos concentrado, bien por la vía de la revolución como en México, o bien por la vía de la política pública, generaron una amplia movilización de colonización periférica que siempre ha ido por delante de la presencia estatal (Palacios, 1995).

La periferia no solo fue geográfica, sino administrativa, y agravada por la violencia ejercida por las luchas entre partidos. En esas áreas de colonización aislada, desde los tiempos coloniales hasta nuestros días, la organización y regulación de las relaciones sociales fue dejada a la libre iniciativa de individuos, grupos sociales y más tarde grupos armados, sin pasar por las instancias estatales. Así, muchas de estas regiones se fueron integrando gradualmente al conjunto de la nación tanto por la expansión de las vías de comunicación como por la integración a la producción nacional (González y Otero, 2006). Encontramos la conjunción de los procesos sociales, las bonanzas económicas emergentes, la violencia, los factores ecológicos, culturales y políticos como el marco en el que el Estado se ha desarrollado a través de una presencia diferenciada, y que ha llevado a que en su seno convivan orden y violencia no sólo como resultados de la presencia estatal sino como las bases constructoras del mismo (González, Bolívar y Vásquez, 2003), el carácter moderno del Estado como tal queda puesta en entredicho ante esta evidencia. 


\section{El Estado como legítimo depositario de la voluntad popular}

El supuesto básico del concepto de la gobernanza es la idea de que unas instituciones estatales fuertes conducen a la canalización de las demandas de la sociedad civil. La gobernanza se consigue mediante la modernización de las prácticas de gobierno democrático, la descentralización de la administración pública, la conversión de los parlamentos en órganos más técnicos y menos políticos, y el control estatal de los medios de comunicación (González y Otero, 2006)

Ahora bien, en el discurso político identitario, se asume la legitimidad y capacidad de gobernanza del Estado basado no en el desempeño universal de sus atributos, sino en el recoger la expresión de la mayoría. El gobierno de Andrés Pastrana inició la negociación con las guerrillas porque el movimiento ciudadano por la paz y la ciudadanía en general urgía una salida negociada al conflicto. Por eso el gobierno, como legítimo representante de la voluntad popular, emplaza a la comunidad internacional a apoyar el deseo popular. Una vez rotas las negociaciones tal como ya se señaló, el presidente aduce que las FARC le han engañado, pero no solo a él, sino a "todos los colombianos", y en función de ese engaño "al pueblo" inicia una segunda fase de la política exterior ya no basada en los diálogos sino en el combate.

Los gobiernos de Álvaro Uribe por su parte se consideran herederos de la voluntad ciudadana de afrontar el problema de la inseguridad, en contraposición al gobierno anterior que se retrata como débil, e incapaz de asumir la voluntad popular de enfrentar a los "enemigos". Además de la elección en primera vuelta en los dos procesos electorales en los que se presentó, el elemento de la "popularidad" se suma a la pretensión de legitimidad. En ese sentido los elementos personales que consiguen la identificación popular con el mandatario entran a formar parte del juego de la "legitimidad del gobierno". Por esto, la autoridad, la cercanía con el pueblo, y los valores representados por el presidente, también juegan un papel importante en la imagen del Estado que se presenta, a través de la cabeza de gobierno, al sistema internacional y en la forma en que las críticas provenientes del exterior al mandatario y su estilo se emplazan como críticas a lo que somos, una diferenciación entre "nosotros" los demócratas y los defensores del terrorismo. Dos buenos ejemplos de esta estrategia se encuentran en la definición por parte del presidente, sobre el "bloque intelectual de las FARC" que hacía referencia a intelectuales y organizaciones que denunciaban en el exterior la connivencia entre el gobierno y el paramilitarismo, o lo que denomina los "enemigos agazapados

\footnotetext{
${ }^{5}$ Presidente Uribe denunció existencia de 'bloque intelectual de las FARC' 07/02/2009 El Tiempo, Sección política.
}

Araucaria. Revista Iberoamericana de Filosofía, Política y Humanidades, año 20, n 39. Primer semestre de 2018. Pp. 491-507. ISSN 1575-6823 e-ISSN 2340-2199 doi: 10.12795/araucaria.2018.i39.23 
de la democracia" que en este caso son otros candidatos a cargos públicos que diferían de su postura.

En el caso del presidente Santos, en su primer mandato se presenta a las elecciones como heredero de Uribe, como una expresión popular de la necesidad de continuar con la Política de Seguridad Democrática. A lo largo del mandato se constituye la posibilidad de dialogo con la guerrilla y usa su candidatura electoral a un segundo periodo como la forma de refrendar dicha propuesta como mandato popular.

Las campañas electorales "monográficas" son una forma de justificar la legitimidad del estado sin entrar en los grandes debates sobre las capacidades, representatividad y justicia del mismo. Por otro lado, aunque no se pone en duda el carácter democrático del régimen colombiano, y por el contrario es importante reseñar la falta de sintonía social de las guerrillas y paramilitares como actores políticos. Es cierto que, dada la ausencia estatal, al constituirse los grupos guerrilleros como la única autoridad histórica en ciertas zonas del país, se han convertido en los voceros de la ciudadanía, bien por ser los únicos agentes de la regulación social o bien a través del clientelismo armado (Cubides, 2005).

La verdad es que el Estado ni opera, ni representa a una importante cantidad de colombianos que viven en la periferia del sistema. De hecho, en algunas zonas del país la entrada del Estado a través del Ejército o en el intento por promover la erradicación voluntaria de cultivos de coca, se ha vivido como un proceso de ocupación con importantes tensiones entre la población y las fuerzas del orden gubernamentales y los representantes institucionales. Todo esto se ve agravado porque el Estado no solo es ilegítimo en algunas zonas sino también inoperante e ineficaz. La escaza sostenibilidad y eficacia de los planes sociales con los que intenta y ha intentado intervenir en estas zonas de "periferia" le granjean un mayor resquemor por parte de los pobladores.

\section{El Estado víctima}

Articulando todos los demás elementos de la auto-representación del Estado, tenemos su constitución en una narrativa como víctima. Esta imagen hace una lectura histórica según la cual la paz no se alcanzaba porque hay unos grupos que no lo permiten (armados y/o políticos) pero, además, no tener paz limita el crecimiento económico. La génesis del Estado a través de su relación con la violencia y su implantación a través de las redes clientelares, la fragmentación del poder regional, etc. se desplazan en la lectura histórica, para

\footnotetext{
6 “"Aún hay enemigos agazapados de la seguridad democrática', afirma el presidente Uribe”, 19/02/2010 El Tiempo, Sección política.
} 
dar lugar a la afirmación: "si no tuviésemos guerra todo sería diferente, todo iría bien".

La paz se constituye en un fin en sí mismo, un estado de cosas en las que el desarrollo, y el bienestar florecerán por sí mismos. La paz con los grupos armados no se entiende como un paso en un proceso sino como el fin. En el ya reseñado discurso del presidente Santos, la Paz es una forma de evitar ser un Estado fallido. Por tanto, como víctima de la guerra, la mayor aspiración del Estado es terminar con su victimario, para terminar así con su problema. En este caso la política reciente hace una distinción entre los anteriores gobiernos y el actual. Antes los victimarios eran los grupos al margen de la ley que atentaban contra el Estado. Tras el proceso de paz, los victimarios son un grupo difuso y heterogéneo de "enemigos de la paz", conformado por sus detractores políticos, los expresidentes Uribe y Pastrana a la cabeza, y después por un conjunto de actores diversos políticos, religiosos y armados. En el primer caso la paz era una idea amplia centrada en la ausencia de guerra y la preminencia por imposición del actor estatal; en el segundo caso, la "paz" cambia de significado para centrarse en dar nombre al resultado del proceso de paz con la guerrilla y la implementación del Acuerdo de la Habana.

El histórico planteamiento del Estado como víctima derivó en una suerte de política de venganza en la que se permitían muchos excesos para combatir al enemigo: desde la justificación de la existencia del paramilitarismo hasta las violaciones de derechos cometidos por miembros de los cuerpos de seguridad del Estado bajo la justificación de la guerra antisubversiva.

La narrativa del Estado víctima, cerraba cualquier posibilidad de que el Estado se juzgara en igualdad de condiciones junto con sus enemigos como un victimario más. Por el contrario, recurría a que se reconociera su indefensión no sólo ante los terroristas sino ante los que se oponen a su política y que juegan sucio mientras el gobierno y las Fuerzas Militares cumplen con unas normas que les dejan estrechos márgenes de acción ${ }^{7}$.

En el marco del proceso de diálogo con la guerrilla se hizo un reconocimiento a la existencia de causas objetivas de la guerra, lo cual queda reflejado en los seis puntos del Acuerdo. Si bien, en el discurso gubernamental no se hace ninguna alusión a que la existencia de la guerrilla estuviera justificada por dichas causas. En los diálogos de La Habana se hacía una mención al conflicto como si fuera un ente existente por encima de los grupos en confrontación, y no un producto de sus acciones. Lógicamente, esto se explica en que una estrategia de culpabilidades cruzadas nunca habría podido llegar a un acuerdo, sin embargo, sí que supone una dificultad en el reconocimiento político del otro guerrillero.

7 31/01/2009 Secretaría de Prensa de la Presidencia de Colombia, Álvaro Uribe: "Fuerzas Armadas de Colombia son las que más esfuerzos hacen por la observancia de los derechos humanos en el mundo" http://web.presidencia.gov.co/sp/2009/enero/31/10312009.html 
El fin último del dialogo fue el paso a la vida política del grupo guerrillero, no obstante, no se le otorgó legitimidad a su origen, es decir, legitimidad de su causa, sino que se aseguran los medios para que pueda defenderla. Sin duda, este es un logro notable del proceso de paz para resolver una situación tan compleja. Asimismo, también supone uno de los mayores desafíos del postconflicto, otorgar garantías y un lugar en el debate político institucional a un actor cuya legitimidad existencial no ha sido reconocida y de hecho es el principal argumento de los detractores del proceso.

Este es el problema fundamental que se recrea en el actual espacio político en Colombia. Los detractores del proceso de paz niegan aun la posibilidad de que las extintas FARC puedan jugar un papel político porque siguen anclados en determinarles como grupo terrorista únicamente. El reconocimiento de beligerancia nunca ha sido completo. El gobierno, por su parte, tendría que ser capaz de promover una construcción discursiva novedosa o distinta de espacio de la participación, si bien esta propuesta ha iniciado con mal pie si se atiende a los enormes fallos de la estrategia de la campaña por el "sí" en el plebiscito por la paz acontecido el 3 de octubre de 2017. Por el contrario, el gobierno actual parece aplicarse más en sacar adelante la implementación del Acuerdo a través de juego político clientelar tradicional. Lo que popularmente se conoce en Colombia como la "mermelada", es decir, las dádivas del presidente hacia los "caciques" políticos que le aseguran su apoyo en el Congreso.

La implementación del Acuerdo de Paz supone, por tanto, un funcionamiento distinto y crítico de la institucionalidad colombiana, que entiende un reconocimiento de los orígenes de la confrontación en las fallas de la política y el sistema de la representación. A pesar de esto, para conseguirlo se redunda sobre las mismas y tradicionales formas cuestionables de la acción política.

\section{EI Estado liberal como referente}

Finalmente reseñar la importancia que en la construcción de la identidad tiene la afinidad a un modelo económico y a una idea del papel del Estado en la provisión del bienestar. La más importante característica de la representación económica es la referencia a un modelo liberal con una importancia superlativa de la inserción en la economía internacional. De hecho, Colombia puede considerarse como uno de los países más liberales de su entorno inmediato. Teófilo Vázquez (2010: 143) afirma que en la Constitución colombiana de 1991 se redefine el marco de las relaciones entre Estado y sociedad, fortaleciendo el carácter social de la acción estatal. Empero, el autor muestra que la situación colombiana está cruzada por una irremediable contradicción. De un lado, 
consagró a Colombia como un Estado Social de Derecho. De otro, y en el contexto del ajuste estructural promovido por agencias internacionales como el Banco Mundial y el Fondo Monetario Internacional durante los 1990 (el llamado Consenso de Washington), el Estado colombiano implementó procesos de privatización y desregulación de actividades económicas y de prestación de servicios públicos. Esto último y el carácter subordinado de Colombia en el orden internacional hace que las preocupaciones por redefinir las relaciones entre gobernantes y gobernados se vean afectadas por la extensión de los mecanismos de mercado a las más diversas relaciones sociales.

Por el otro lado, la crisis económica de final de la década de los 1990 y la emergencia de una política de seguridad totalizante conllevan un fortalecimiento de la idea de que la "salvación de la economía nacional está en el sistema internacional"; lo que el Gobierno de Álvaro Uribe definirá en buena parte bajo su idea de mejorar la "confianza inversionista". En este sentido la política económica exterior y su vínculo con la seguridad encajan en las características de lo que algunos teóricos llaman el enfoque de "seguridad de la economía". En su caso el Presidente Santos planteó en su primer gobierno un modelo económico para el país basado en las "cinco locomotoras" a saber: vivienda, minería, infraestructuras, agricultura e innovación (DNP, 2010). En su segundo mandato reformuló el Plan Nacional de Desarrollo 2014-2018 basándolo en unas premisas más sociales: paz, equidad y educación, aunque en el fondo del documento subyace el mismo modelo económico (DNP 2014-2018).

Por supuesto, la formulación de las "locomotoras" como tal es demasiado genérica, pero su desarrollo en el marco de las políticas públicas no deja duda de que los patrones del modelo liberal se mantienen. El objetivo es en todas estas áreas crear y asegurar los incentivos para la inversión extranjera. La paz sigue considerándose, por ende, como lo ha hecho en los últimos treinta años, una fórmula para conseguir la estabilidad que atraiga los capitales extranjeros y no una fórmula para conseguir una mejor y más justa distribución de los recursos en uno de los países más desiguales del mundo.

El modelo de la seguridad económica, desarrollado en la Postguerra Fría, busca la salvaguarda de la integridad estructural de las capacidades para generar prosperidad y los intereses en general de una entidad político-económica en el contexto de los variados riesgos y amenazas que enfrenta en el sistema económico internacional (Dent, 2010).

En el caso colombiano sus intereses se definen también en convertirse en un destino seguro para las inversiones de otros. Como una economía liberalizada que es en términos legales e institucionales requiere generar condiciones internas que permitan su inserción en el sistema económico globalizado. Este

${ }^{8}$ Uribe, A: Confianza Inversionista, sin fecha, en su página web: http://www.alvarouribevelez. com.co/es/content/confianza-inversionista

Araucaria. Revista Iberoamericana de Filosofía, Política y Humanidades, año 20, n 39. Primer semestre de 2018. Pp. 491-507. ISSN 1575-6823 e-ISSN 2340-2199 doi: 10.12795/araucaria.2018.i39.23 
hecho es muy importante como componente de la identidad estatal; pues las condiciones de política exterior y los actores con los que se estrechan las relaciones diplomáticas serían muy diferentes si el Estado colombiano se definiera como proteccionista o como una economía social, como por ejemplo se ha definido Bolivia. Asimismo, esta vocación liberal genera una de las mayores inconsistencias y riesgos en la implementación del Acuerdo de Paz.

El Acuerdo supone la puesta en marcha de importantes programas para desarrollar el campo, históricamente olvidado, así el gobierno intenta entrar a los territorios ofreciendo programas que en esencia se basan en buscar la substitución de cultivos ilícitos. Sin embargo, sus "macro-políticas" en este sector o en la minería van dirigidas a la gran explotación agrícola. Dado que el problema esencial de Colombia es la concentración de la tierra productiva en pocas manos y la pobreza general de la población rural estas dos políticas enfrentadas o bien ponen en entredicho la voluntad real del gobierno de materializar la paz, o bien se convierte en una bomba de relojería que puede agravar la confrontación social. Parece, a tenor de las recientes tensiones con comunidades productores de coca que recelan del gobierno y su propuesta de sustitución, y ante las movilizaciones indígenas, que la segunda alternativa es la más plausible ahora mismo.

\section{Conclusiones}

A lo largo de este artículo se han analizado los cuatro pilares de la autorreferencia del Estado desde el discurso gubernamental. La identidad juega un papel clave en la definición de las políticas y de las posturas que toman las elites en la discusión con otros actores políticos. Colombia no es una excepción y en un proceso de superación del histórico conflicto armado con las guerrillas, buena parte de los preceptos identitarios del Estado deberían ser objeto de crítica. En primer lugar, porque el colombiano ha sido un Estado construido de forma paralela y estrechamente relacionada con el desarrollo de la violencia. En segundo lugar, porque el proceso de paz supuso un importante esfuerzo de reconocimiento y relato de las debilidades del Estado producto de varias de sus características identitarias como, por ejemplo, su identificación con el modelo liberal económico; o su reivindicación de calidad institucional legitimidad democrática a pesar de su estructura fragmentada o difusa y de la histórica desconexión con amplias regiones del país.

Bien es cierto que en el gobierno de Santos se han producido cambios importantes en el discurso político que reflejaron la oportunidad para entablar el diálogo y tal como ocurrió alcanzar un Acuerdo. Un proceso duro y muy difícil que no puede desaprovecharse. 
Dentro de los cambios del discurso más importantes formulados por el Presidente Santos está el reconocimiento de la confrontación paso indispensable para la negociación, y más importante aún la voluntad de llegar a un acuerdo para que las FARC participen en política. También el reconocimiento de las víctimas, incluso las del Estado y en especial de la necesidad de reparación y restauración de la memoria, además del reconocimiento de la paz como un proceso de construcción y no como un resultado de la imposición. No obstante, a pesar de los cambios muy relevantes en lo que supone el tratamiento del conflicto, estos no han alterado ni generado una discusión dentro de las elites suficientemente seria y profunda sobre las creencias en las que se funda la definición del modelo político, social económico que caracteriza al país y que define sus políticas. En este artículo se han identificado tres discursos básicos que articulan la definición del Estado: 1) Colombia cuenta con un sistema institucional completo y autosuficiente; 2) el Estado es depositario de la voluntad popular y por tanto legítimo; y 3) finalmente, los fallos del Estado y deficiencias de la economía son causadas porque el Estado es una víctima de actores ajenos y arrogantes que no le han permitido desarrollarse y ejercer correctamente su papel.

Pero lo cierto es que estas premisas no se ajustan del todo a la realidad dado que Colombia no controla la totalidad de su territorio y aún tiene importantes problemas para alcanzar dicho control (Ríos, 2017). Además, Colombia no es un país donde la participación política sea suficientemente fuerte, los mecanismos disfuncionales de la política siguen siendo los que determinan la forma de hacer política y finalmente porque la idea de que el Estado ha sido una víctima de actores arrogantes y violentos no es del todo cierta. El Estado en Colombia es también un actor violento que se ha valido del uso de la de la cohesión y de otras formas de fuerza para aplacar opositores y movilizaciones sociales. Es decir, no es exclusivamente una víctima y, en cualquier caso, su papel de víctima no sirve como justificación de sus falencias, esto se hace más relevante aun ante la desaparición de uno de los actores en confrontación.

Finalmente, el artículo también analiza una de las características definitorias de la política del Estado, su adhesión al modelo económico liberal. El modelo dominante en la política económica y social entra en conflicto con el modelo de economía social que se necesitaría para conseguir superar el problema de la pobreza y baja productividad agrícola y, cómo no, el de los cultivos ilícitos.

Acabar con la guerra y asumir la paz dotándola de un significado que incluya a todos los colombianos supone repensar y reformular discursivamente la forma en la que se considera la identidad del Estado, sus preceptos básicos y la construcción de un orden social y un status quo. La paz entra en conflicto con los preceptos fundamentales de una construcción estatal elitista y cerrada que se articuló excluyendo del debate a otras visiones ideológicas. 


\section{Referencias bibliográficas:}

07/02/2009 E1 Tiempo, Sección política: Presidente Uribe denunció existencia de 'bloque intelectual de las FARC'

19/02/2010 El Tiempo, Sección política. ““Aún hay enemigos agazapados de la seguridad democrática', afirma el presidente Uribe”,

21/11/2014 Revista Semana; Santos: "Colombia es hoy un país líder que evitó ser un Estado fallido" Disponible en: http://www.semana.com/nacion/ articulo/juan-manuel-santos-en-foro-en-estados-unidos-colombia-es-hoyun-pais-lider-que-evito-ser-un-estado/411900-3

22/04/2017 El Tiempo: Presidente Santos respondió las críticas de Nicolás Maduro

24 /11/2016 Insight Crime: Santos reconoce que grupos criminales se están trasladando a áreas de las FARC; Disponible en: http://es.insightcrime. org/analisis/santos-reconoce-grupos-criminales-estan-trasladando-areasfarc

31/01/2009 Secretaría de Prensa de la Presidencia de Colombia: Álvaro Uribe: "Fuerzas Armadas de Colombia son las que más esfuerzos hacen por la observancia de los derechos humanos en el mundo" http://web.presidencia. gov.co/sp/2009/enero/31/10312009.html

Bejarano, A., y Pizarro, E. (2003). "Colombia: The Partial Collapse of the State and the Emergence of Aspiring State-Makers". En P. Kingston e I. Spears (eds.) States Within States: Incipient Politial Entitites in the Post-Cold War Era (99-118). Nueva York, Pallgrave McMillan;

Borda, S. (2012). La internacionalización de la paz y de la guerra en Colombia durante los gobiernos de Andrés Pastrana y Álvaro Uribe. Búsqueda de la legitimidad politica y capacidad militar. Bogotá: Norma

Campbell, D. (1998) Writing Security. United States and the politics of identity Manchester: Manchester University Press

Cubides, F. (2005). Burocracias Armadas. Bogotá: Norma. DEPARTAMENTO NACIONAL DE PLANEACION. (2010). Prosperidad para todos, Plan Nacional de Desarrollo 2010-2014. Bogotá: DNP

DEPARTAMENTO NACIONAL DE PLANEACION (2014). Todos por un nuevo país, Plan Nacional de Desarrollo 2014-2018. Bogotá: DNP.

Duncan, G. (2006). Los señores de la guerra. Bogotá: Planeta.

Echandía, C (2006). Dos décadas de escalamiento del conflicto armado en Colombia 1986-2006. Bogotá: Universidad Externado.

Gilhodes, P. (1972) Las luchas agrarias en Colombia. Bogotá: La Carreta. Gómez, F., Wills, M. et al. (2004). Nuestra guerra sin nombre: Transformaciones del conflicto en Colombia. Bogotá: IEPRI 
González, F; Bolívar, I; y Vásquez, T. Vazquez, T. (2003). Violencia política en Colombia. De la nación fragmentada a la construcción del Estado. Bogotá: CINEP.

González, F y Otero, S. (2006). La presencia diferenciada del Estado: un desafio a los conceptos de gobernabilidad y gobernanza. Institut de recherche et debat sur la governance http://www.institut-gouvernance.org/fr/analyse/ fiche-analyse-237.html

Gutiérrez, F. (2014). El orangután con sacoleva. Cien años de democracia y represión en Colombia (1910-2010). Bogotá: Debate.

Hansen, L. (2006). Security as Practice: Discourse Analysis and the Bosnian War. Londres: Routledge.

López, C. (2016). ¡Adios a las Farc! ¿ya hora qué? Bogotá: Debate

Mason, A. (2002). "Exclusividad, autoridad y Estado". Análisis Político, 47, 5- 75.

Oquist, P. (1978). Violencia, conflicto y política en Colombia. Bogotá: Instituto de Estudios Colombianos.

Palacios, M. (1995). Entre la legitimidad y la violencia. Colombia 1875-1994. Bogotá: Norma.

Pécaut, D. (2006). Crónica de cuatro décadas de política colombiana. Bogotá: Norma.

Ramírez, W. (1990). Estado, violencia y democracia. Bogotá: Universidad Nacional.

Reyes, A. (1988). "Conflictos agrarios y luchas armadas en la Colombia contemporánea: una visión geográfica”. Análisis Político, 5, 7-29.

Ríos, J. (2017) "Determinantes geográfico-políticos de la acción violenta guerrillera: un análisis de la concurrencia regional de guerrillas y paramilitares en el conflicto colombiano". Revista Española de Ciencia Politica, 44, 121-149.

Rodríguez, E. (2015) "Narcotráfico y conflicto armado en Colombia". Ibañez, F. et al. Mercados ilegales y violencia armada. Barcelona: Tecnos

Rodríguez, E. (2017). "Internacionalización del Conflicto, del Acuerdo y del Postacuerdo: Evolución, Continuidades y Rupturas de una Estrategia”. Análisis Político, 30 (90), 194-208.

Sanahuja, J. y Shunemann,J. (2010). SANAHUJA, J; SCHÜNEMANN, J (2010) "El Nexo Seguridad-Desarrollo: entre la Construcción de la Paz y la Securitización de la Ayuda”. Sanahuja, J. (Coord.). Construcción de la paz, seguridad y desarrollo. Visiones, políticas y actores; Madrid: Editorial Complutense, ICEI. 
Tickner, A (2001). "La guerra Contra las Drogas: Las Relaciones ColombiaEstados Unidos Durante la Administración Pastrana”. En Estrada, J (ed.). Plan Colombia: Ensayos críticos. Bogotá: Universidad Nacional de Colombia.

Tokatlian, J. G. 1 (2008) "La construcción de un "Estado fallido" en la política mundial". Análisis Político, 64, 67-104.

Uribe, A (s-f-) Confianza Inversionista, sin fecha, en su página web: http:// www.alvarouribevelez.com.co/es/content/confianza-inversionista

Vargas, R. (1999) Drogas, máscaras y juegos. Narcotráfico y conflicto armado en Colombia. Bogotá: Tercer Mundo.

Vásquez, T (2010). “La Constitución del 91: entre el reconocimiento de los derechos sociales y el modelo de desarrollo". En Launay-Gama, C. y González, FGobernanza y conflicto en Colombia. Interacción entre gobernantes y gobernados en un contexto violento. Bogotá: Editorial Pontificia Universidad Javeriana. 
